\title{
Medienunterstützter Wortschatzerwerb am Beispiel des Italienischen
}

\author{
Ass.-Prof. ${ }^{\text {in }}$ MMag. ${ }^{\text {a }}$ Dr. ${ }^{\text {in }}$ Eva Maria Hirzinger-Unterrainer \\ Institut für Fachdidaktik, Bereich Didaktik der Sprachen \\ Universität Innsbruck
}

\section{Einleitung}

Der vorliegende Beitrag beschäftigt sich mit dem Wortschatzerwerb im Rahmen des schulischen Italienischunterrichts und verweist auf eine empirisch untersuchte Möglichkeit, wie dieser durch Nutzung verschiedener Medien gefördert werden kann. In Hinblick auf den Erwerb der lexikalischen Kompetenz im Allgemeinen ist festzuhalten, dass diesem innerhalb der Forschung bis in die 1980er Jahre nur eine marginale Rolle zukam (Nation 2011: 529). Erst in den 1990er Jahren entwickelte sich der Bereich zu einer ,booming area in linguistic research and language teaching practice“ (Read 2000: 251), allerdings gilt dies nur bedingt für das Italienische ${ }^{1}$, da hier auch aktuell noch ein eher geringes Interesse am Wortschatzerwerb festgestellt wird: „A number of studies in this category have sought to trace the acquisition or development of features of the Italian language, mostly its morphosyntax. In fact, there has been very little interest in the acquisition of Italian VOCABULARY since the year 2000.” (Macaro 2010: 130; vgl. Hirzinger-Unterrainer 2014).

Um diesem Forschungsdesiderat entgegenzuwirken, wird nachfolgend eine work-in-progress Studie präsentiert, die sich mit dem medienunterstützen Wortschatzerwerb des Italienischen beschäftigt. Dazu wird eingangs ein kurzer Einblick in die bisherige Forschung gegeben, um im Anschluss erste Ergebnisse der Studie zu präsentieren.

1 Der Großteil der Forschung zum Wortschatzerwerb beschäftigt sich mit Englisch als Zielsprache (vgl. u.a. Schmitt 2010: 28). 


\section{Medienunterstützer Wortschatzerwerb}

Die digitalen Medien bilden einen wichtigen Bestandteil unseres täglichen Lebens, was sich allein darin zeigt, dass der Besitz und die Nutzung digitaler Medien ständig zunehmen. Für Kinder und Jugendliche im deutschen Sprachraum wird dieser Umstand jährlich in der sogenannten KIM- bzw. JIM-Studie abgebildet (mpfs 2015). Für die JIM-Studie 2015 wurde in Deutschland eine Stichprobe von 1.200 Jugendlichen im Alter von 12 bis 19 Jahren im Zeitraum von Mai bis Juni 2015 telefonisch befragt. In Bezug auf die Medienausstattung gilt festzuhalten, dass gemäß JIM-Studie 2015 so gut wie alle Jugendlichen zwischen 12 und 19 Jahren in Haushalten mit Fernseher, Computer, Internetzugang und Handys aufwachsen (mpfs 2015: 6): 99,0\% der Haushalte verfügen über ein Handy, 98,0\% über einen Computer/Laptop, 97,0\% über ein Fernsehgerät und $96,0 \%$ über einen Internetzugang. Mit je 86,0\% erfreuen sich auch die Digitalkamera und das Radiogerät großer Beliebtheit. Was den Gerätebesitz der Jugendlichen betrifft, geben 98,0\% an, über ein eigenes Handy zu verfügen, 58,5\% besitzen einen eigenen MP3-Player und 76,0\% einen Computer (mpfs 2015: 8). Es erscheint daher naheliegend, diesen Umstand gemäß mobilen Lernens für das Sprachenlernen, und hier v.a. für das Wortschatzlernen, nutzbar zu machen (vgl. Unterrainer 2012: 28).

Im Unterricht im Allgemeinen sowie im Fremdsprachenunterricht im Speziellen haben die digitalen Medien in Österreich in geringerem Ausmaß Einzug gehalten. Nach wie vor bilden überwiegend die traditionellen Medien wie Tafel und Overheadprojektor die Hauptstütze des Fremdsprachenunterrichts wie etwa die teilnehmende Beobachtung am für die vorliegende Studie untersuchten Italienischunterricht zeigt (vgl. Hirzinger-Unterrainer i.V.).

Bisherige Studien zum Fremdsprachenlernen mittels digitaler Medien fokussieren das mobile Lernen. Ein Begriff, der im deutschsprachigen Raum meist „Lernen mit mobilen Endgeräten und Softwareanwendungen“ (Hug 2010: 195) umfasst und synonym mit M-Learning und Mobile Learning verwendet wird. Mit anderen Worten, ,'mobile learning' is frequently used to refer to the use of handheld technologies enabling the learner to be 'on the move', providing anytime anywhere access for learning“ (Price 2007: 33f.). In diesem Sinne können die

Barbara Hinger (Hg.), Zweite „Tagung der Fachdidaktik“ 2015.

Sprachsensibler Sach-Fach-Unterricht - Sprachen im Sprachunterricht.

(C) 2016 innsbruck university press, ISBN 978-3-903122-51-2, DOI 10.15203/3122-51-2 
Lernenden mithilfe mobiler Geräte fast überall und jederzeit lernen (vgl. Unterrainer 2012: 24) und damit etwa mögliche Warte- oder Fahrtzeiten für das Lernen nutzen.

In den bislang vorgelegten Studien wurde das medienunterstützte Lernen überwiegend mit Studierenden und damit mit jungen Erwachsenen untersucht. So legen Kennedy \& Levy (2008) eine Studie zum Effekt von Lernen italienischer Vokabeln via SMS vor. Dazu haben sie in Australien Studierenden eines Italienisch-Kurses für AnfängerInnen regelmäßig SMS mit Vokabeln, Grammatik und kursrelevanten Informationen geschickt. Sie konstatieren eine Erweiterung und Festigung des italienischen Wortschatzes durch das Lernen via SMS, das jedoch nicht anhand eines Wortschatztests gemessen wurde, sondern auf einer quantitativen Selbsteinschätzung mittels einer schriftlichen Befragung durch die Studierenden basiert. Des Weiteren beinhalteten die SMS neben Wortschatz auch Grammatik und kursrelevante Informationen, wodurch nicht (nur) das Konstrukt, Wortschatz' überprüft wurde (vgl. Hirzinger-Unterrainer 2014).

Eine weitere Studie, allerdings für Englisch als Fremdsprache, ist jene von Hasegawa, Ishikawa, Shinagawa, Kaneko \& Miyakoda (2008), die mit SIGMA (Special-Interested-Group Material Accumulator) ein speziell konzipiertes Vokabellernsystem geschaffen haben. Japanische Lernende kreierten individuell ihre Materialien für das Lernen von englischen Vokabeln, indem sie Bilder zur Erstellung eigener, auf fünf Sekunden pro Wort beschränkte, Videos erstellten. Jedes dieser Videos beinhaltet Bedeutung und Aussprache des zu lernenden Wortes und kann auf mobilen Geräten abgespielt werden. Die Studierenden, die mit SIGMA lernten, erreichten eine größere Behaltensrate der Vokabeln, allerdings sind auch hier Einschränkungen der durchgeführten Studie zu nennen, da diese nur mit zehn Versuchspersonen durchgeführt wurde, die wiederum lediglich zwanzig Wörter lernten.

Eine weitere Möglichkeit, Vokabeln medial zu lernen, zeigen Joseph, Binsted \& Suthers (2005) auf, indem durch Kameras erstellte Bilder (PhotoStudy) verwendet werden. Dadurch konnten die Lernenden Wort und Bild-Verknüpfungen mit der Aussprache lernen (vgl. Hirzinger-Unterrainer 2014).

Thornton \& Houser (2005) versendeten in ihrem Projekt Learning on the MoveLOTM an 44 japanische Englisch-Lernende E-Mails (beschränkt auf 100 Wör-

Barbara Hinger (Hg.), Zweite „Tagung der Fachdidaktik“ 2015.

Sprachsensibler Sach-Fach-Unterricht - Sprachen im Sprachunterricht.

(C) 2016 innsbruck university press, ISBN 978-3-903122-51-2, DOI 10.15203/3122-51-2 
ter) mit Informationen zu Vokabeln, wobei die Lernenden diese Informationen gemäß des Push-Prinzips erhielten, d.h. sie bekamen diese ohne selbst die Initiative ergreifen zu müssen ${ }^{2} .71,0 \%$ dieser Lernenden bevorzugten es, die Lektionen über das Handy - und nicht über den Computer - abzurufen. Zudem konnten sich $91,0 \%$ vorstellen, diese Lernmethode weiterhin zu nutzen (vgl. HirzingerUnterrainer 2014).

Eine weitere Studie mit japanischen Studierenden legte Stockwell (2010) vor. Diese wurde in drei Kohorten (in den Jahren 2007, 2008 und 2009) mit insgesamt 175 Englisch-Lernenden zum Unterschied des Vokabellernens mit mobilen oder Stand-Geräten durchgeführt. Die kursbegleitenden Aktivitäten für den Wortschatzerwerb wurden auf einer Plattform (Moodle) zur Verfügung gestellt, die über mobile Geräte oder über den PC genutzt werden konnte. Zwischen den beiden Varianten konnten keine signifikanten Unterschiede bei den richtigen Antworten festgestellt werden. Der Zeitaufwand für die Durchführung der Aktivitäten ist jedoch bei der mobilen Version deutlich höher, was Stockwell u.a. auf den kleineren Bildschirm und die kleine Tastatur zurückführt. Über die Hälfte der Studierenden griff nie auf das Smartphone zurück, um den Wortschatz zu üben; allerdings zeichnete sich für das Jahr 2009 eine steigende Nutzung der mobilen Version ab.

Wie aus den hier knapp dargestellten Studien ersichtlich wird, wurde überwiegend der Wortschatzerwerb in der Zielgruppe Studierende untersucht. Gerade für den Bereich der Fremdsprachendidaktik erscheint es jedoch erstrebenswert, auch SchülerInnen zu beforschen, um zielgruppenadäquate Forschungsergebnisse direkt in den schulischen Fremdsprachenunterricht einfließen zu lassen. Insgesamt ist ebenfalls ein Forschungsdesiderat hinsichtlich (Longitudinal-)Studien mit anderen Sprachen als Englisch auszumachen. Nachfolgend werden erste Ergebnisse einer Studie der Autorin präsentiert, die das medienunterstützte Lernen des italienischen Wortschatzes von SchülerInnen an einer österreichischen Allgemeinbildenden Höheren Schule (AHS) in den Blickwinkel nimmt und damit versucht, den angeführten Forschungsdesiderata zu begegnen.

2 Zu einer umfassenderen Darstellung des Push- und Pull-Prinzips im Zusammenhang mit neuen Medien bzw. Programmen s. etwa Wienold (2004).

Barbara Hinger (Hg.), Zweite „Tagung der Fachdidaktik“ 2015.

Sprachsensibler Sach-Fach-Unterricht - Sprachen im Sprachunterricht.

(C) 2016 innsbruck university press, ISBN 978-3-903122-51-2, DOI 10.15203/3122-51-2 


\section{Forschungsdesign}

In der vorliegenden Studie wurden den teilnehmenden SchülerInnen alternative Möglichkeiten aufgezeigt, den Italienisch-Wortschatz zu lernen. Dazu nahmen im Schuljahr 2012/13 113 SchülerInnen, die sich im ersten, dritten und vierten Lernjahr Italienisch befanden, am Projekt teil. Das Forschungsinteresse bezog sich darauf, mögliche Unterschiede festzustellen zwischen Lernenden, die den Wortschatz mit Hilfe von digitalen Medien lernten (Experimentalgruppe), und jenen, die sich diesen auf ,herkömmlichem` Wege aneigneten (Kontrollgruppe). Diese Unterschiede wurden einerseits im Wortschatzerwerb und andererseits in den verwendeten Vokabellernstrategien angenommen. Die Studie umfasste den Zeitraum eines Schuljahres und versuchte unter anderem die nachfolgenden Forschungsfragen $^{3}$ zu beantworten:

(1) Welche Vokabellernstrategien verwenden SchülerInnen im österreichischen Schulkontext?

(2) Welche Unterschiede hinsichtlich der Wortschatzgröße zeigen sich zwischen SchülerInnen, die den italienischen Wortschatz mit digitalen Medien lernen (Experimentalgruppe), und jenen, die diesen ohne digitale Medien lernen (Kontrollgruppe)?

(3) Welche Unterschiede in den Vokabellernstrategien zeigen sich zwischen SchülerInnen, die den italienischen Wortschatz mit digitalen Medien lernen (Experimentalgruppe), und jenen, die diesen ohne digitale Medien lernen (Kontrollgruppe)?

(4) Gibt es darüber hinaus Unterschiede in der Verwendung von Strategien in Bezug auf das Lernjahr (1., 3., 4. Lernjahr; AHS, Sekundarstufe II)?

Zur Beantwortung dieser Fragen bedient sich die Studie einer überwiegend quantitativen aber auch qualitativen Forschungsmethodik. Der quantitativen Forschungsmethodik sind die schriftlichen Vokabeltests (Laufer \& Goldstein 2004 und DIALANG o.J.) zuzuordnen sowie die großteils mit geschlossenen Fragen

3 Die Auflistung sämtlicher Fragestellungen findet sich in Hirzinger-Unterrainer (i.V.).

Barbara Hinger (Hg.), Zweite „Tagung der Fachdidaktik“ 2015.

Sprachsensibler Sach-Fach-Unterricht - Sprachen im Sprachunterricht.

(C) 2016 innsbruck university press, ISBN 978-3-903122-51-2, DOI 10.15203/3122-51-2 
versehene schriftliche Befragung. Die abschließende mündliche Befragung mit ausgewählten Schülerinnen und Schülern ist im qualitativen Forschungsparadigma verortet. Im vorliegenden Beitrag wird ausschließlich auf die Ergebnisse der schriftlichen Befragung über Vokabellernstrategien eingegangen; damit wird hier eine erste Antwort auf die Forschungsfragen (1), (3) und (4) gegeben.

Zeitlich erstreckte sich die Studie, wie bereits erwähnt, über das Schuljahr 2012/13 und bediente sich zweier Datenerhebungszeitpunkte: die teilnehmenden Schülerinnen und Schüler nahmen am Schuljahresbeginn (Oktober 2012) und am Schuljahresende (Juni 2013) an einer schriftlichen Befragung sowie an schriftlichen Wortschatztests teil. Um den Rücklauf des Fragebogens sowie möglichst reliable Ergebnisse der Tests zu gewährleisten, wurde - mit dem Einverständnis der Lehrpersonen und der Schulleitung - eine Paper-Pencil-Befragung in den jeweiligen Unterrichtsstunden aus Italienisch gewählt. Nachfolgend wird die Forschungsmethodik näher beschrieben:

- Schriftliche Paper-Pencil-Befragung über Vokabellernstrategien für Italienisch (vgl. Neveling 2004)

Zu Schulbeginn wurde den SchülerInnen die umfassende Version des Fragebogens mit vier Seiten vorgelegt, die zu Schulende auf drei Seiten gekürzt wurde. Die Version zu Schulbeginn gliedert sich in die Teile „Arbeits- und Lernorganisation“ und „Lernstrategien“. Daran schließen die zwei offenen Fragen „Wie unterscheidet sich das Vokabellernen in den von dir gelernten Sprachen (wie Englisch, Italienisch, ...)?“ und „Wie lernst du Italienisch-Vokabeln am besten?““ an, denen wiederum vier auf einer Skala von 1 bis 10 zu bewertende Aussagen zu Vokabellernen folgen. Der Abschluss des Fragebogens wird durch die „Persönlichen Angaben“ gebildet, die sich aus Geschlecht, Schulstufe, Alter, Muttersprache(n), gelernten Sprachen, Ausbildung der Eltern, Einstellung zur italienischen Sprache und Medienbesitz zusammensetzen. Da die Angaben zur Person häufig eher ungern beantwortet werden und für die befragten Personen nur von geringem Interesse sind, wurden sie an das Ende des Fragebogens gesetzt (vgl. Porst 2008: 143). Des Weiteren nimmt mit der Länge des Fragebogens - gerade bei SchülerInnen - auch die Konzentration ab, wodurch sich die

Barbara Hinger (Hg.), Zweite „Tagung der Fachdidaktik“ 2015.

Sprachsensibler Sach-Fach-Unterricht - Sprachen im Sprachunterricht.

(C) 2016 innsbruck university press, ISBN 978-3-903122-51-2, DOI 10.15203/3122-51-2 
demographischen Fragen am Schluss des Fragebogens anbieten (vgl. Dörnyei 2003). Mit dem ersten Teil des Fragebogens „Arbeits- und Lernorganisation“ sollen die Präferenzen für das Lernen im Allgemeinen in Erfahrung gebracht werden, während der zweite Teil die für den italienischen Wortschatz verwendeten Lernstrategien abfragt. Dadurch sollen die im vorliegenden Beitrag diskutierten Hypothesen „Es besteht ein Unterschied in Vokabellernstrategien zwischen der Experimental- und Kontrollgruppe“ sowie „Es gibt einen Unterschied in der Verwendung von Vokabellernstrategien zwischen SchülerInnen unterschiedlicher Lernjahre (1. oder 3. Lernjahr; AHS, Sekundarstufe II)“ überprüft werden. Für die weitere statistische Auswertung sind diese jeweils zu unterteilen in eine Null- und Alternativhypothese, wobei die Nullhypothese - im Gegensatz zur Alternativhypothese - besagt, dass es die oben angeführten Unterschiede zwischen den unterschiedlichen Gruppen von SchülerInnen nicht gibt.

- Schriftliche Wortschatztests für Italienisch (nach Laufer \& Goldstein 2004 und DIALANG o.J.)

Um Unterschiede im erworbenen Wortschatz zwischen Experimental- und Kontrollgruppe zu erheben, wurden zu Schulbeginn und -ende jeweils zwei Wortschatztests durchgeführt, jener nach Laufer \& Goldstein (2004) sowie jener von DIALANG (o.J.). Der von Laufer \& Goldstein (2004) konzipierte Wortschatztest wurde für das Italienische adaptiert, indem Wörter des Italienischen entsprechend der Frequenz (Intronati 1992) ausgewählt wurden. Die Anordnung der Wörter im Test erfolgte entsprechend ihrer Frequenz, beginnend mit den höherfrequenten Wörtern. Sowohl zu Schulbeginn als auch zu Schulende wurden die Wörter in beide Sprachrichtungen (Deutsch-Italienisch, Italienisch-Deutsch) abgefragt. Damit wurde der Active Recall, bei dem die L1-Übersetzung vorgegeben und die L2-Entsprechung angegeben werden muss, und der Passive Recall, bei dem die vorliegende L2-Entsprechung um die L1 ergänzt werden muss, ebenfalls nach Laufer \& Goldstein (2004: 406), angewandt. Der Test von DIALANG wurde direkt von der online verfügbaren Version übernommen und auf Papierversion gebracht, die von den SchülerInnen im Klassenzimmer ausgefüllt wurden. Im vorliegenden Beitrag wird nicht, wie bereits erwähnt, weiter auf die Wortschatz-

Barbara Hinger (Hg.), Zweite „Tagung der Fachdidaktik“ 2015.

Sprachsensibler Sach-Fach-Unterricht - Sprachen im Sprachunterricht.

(C) 2016 innsbruck university press, ISBN 978-3-903122-51-2, DOI 10.15203/3122-51-2 
tests und deren Ergebnisse eingegangen. Die durchgeführten Wortschatztests sowie die begründeten Ausführungen dazu finden sich ebenso wie deren Ergebnisse in Hirzinger-Unterrainer (i.V.).

- Quasiexperimentelle Untersuchung

Die quasiexperimentelle Untersuchung (Bortz \& Döring 2009: 551) bildet den Kern der vorliegenden Studie. Diese wurde gewählt, da Italienischlernende im schulischen Kontext, und damit „real existierende Populationen“ (Bortz \& Döring 2009: 551), im Forschungsinteresse stehen. Die daran teilnehmenden SchülerInnen untergliedern sich, wie bereits erwähnt, in Experimental- und Kontrollgruppe, die sich zu Schulbeginn wie folgt zusammensetzen:

- Experimentalgruppe

1. Lernjahr Italienisch (5. Klasse AHS): 18 SchülerInnen

3. Lernjahr Italienisch (7. Klasse AHS): 24 SchülerInnen

4. Lernjahr Italienisch (8. Klasse AHS): 14 SchülerInnen

Gesamt: 56 SchülerInnen

- Kontrollgruppe

1. Lernjahr Italienisch (5. Klasse AHS): 24 SchülerInnen

3. Lernjahr Italienisch (7. Klasse AHS): 18 SchülerInnen

4. Lernjahr Italienisch (8. Klasse AHS): 15 SchülerInnen

Gesamt: 57 SchülerInnen

Das experimentelle Design sah vor, dass die Experimentalgruppe den im Unterricht behandelten Wortschatz mittels selbsterstellter Medien lernt, die von den SchülerInnen unter Anleitung der Autorin produziert wurden. Die Kontrollgruppe erwarb den Wortschatz - unbeeinflusst von der Autorin - in der für sie gewohnten Art und Weise, d.h. v.a. durch das Lernen von zweisprachigen Listen, von denen sie eine Spalte abdeckten und die verdeckten Wörter mündlich wiederholten; 86,5\% der Kontrollgruppe verwendeten diese Vorgangsweise zur Wiederholung und Konsolidierung des Wortschatzes zu Studienbeginn. Demge-

Barbara Hinger (Hg.), Zweite „Tagung der Fachdidaktik“ 2015.

Sprachsensibler Sach-Fach-Unterricht - Sprachen im Sprachunterricht.

(C) 2016 innsbruck university press, ISBN 978-3-903122-51-2, DOI 10.15203/3122-51-2 
genüber erstellten die SchülerInnen der Experimentalgruppe in Kleingruppen jeweils ein Medium, das der ganzen Lerngruppe über eine externe Seite (Google Site) und einen universitätseigenen Videostreamer wöchentlich zur Verfügung gestellt wurde. Zu Schulbeginn wurden die SchülerInnen von der Autorin während der Italienisch-Stunde gebeten, sich in Kleingruppen von je drei Personen zu formieren und in eine Liste für einen Termin ihrer Wahl einzutragen, zu dem sie an den universitären Arbeitsplatz der Autorin kommen sollten, um gemeinsam mit ihr den im Unterricht besprochenen Wortschatz anhand eines Mediums aufzubereiten. Dazu standen die folgenden Medien zur Auswahl:

- Audiopodcast bzw. Voki: der zu lernende Wortschatz wird einsprachig auf Italienisch erklärt und anschließend auf der Webseite voki.com eingegeben, wodurch eine Art Audiopodcast entsteht, der online angehört werden kann. Der Vorteil - im Gegensatz zu einem zur Gänze eigenständig produzierten Audiopodcast - liegt in der fast muttersprachlich klingenden Aussprache, die so generiert wird.

- Videopodcast: der Wortschatz wird anhand eines Bildes visualisiert, wobei dieser zusätzlich in Form eines Satzes (auf Italienisch und Deutsch) mit einem Untertitel versehen wird. Als Basis für die Videopodcasts werden im Sinne eines rechtlich korrekten Vorgehens Creative Commons lizenzierte Bilder verwendet, wodurch die SchülerInnen zusätzlich für die Problematik des Urheberrechts sensibilisiert werden.

- Video: die SchülerInnen stellen den Wortschatz durch Körpereinsatz szenisch dar, was von der Autorin gefilmt wurde. Anschließend wurden die einzelnen Szenen mit Hilfe von Windows Movie Maker ${ }^{\circledR}$ mit Untertiteln (italienisches Wort bzw. Wendungen mit deutscher Übersetzung) versehen.

- Wiki: der Wortschatz wird in Form eines Textes als Wiki aufbereitet, in dem unbekannte Wörter auf Italienisch erklärt und/oder anhand eines Bildes dargestellt werden. Diese Variante wurde häufig für das 4. Lernjahr gewählt, da die SchülerInnen im Unterricht entsprechende Texte bearbeiteten und daher den Wortschatz benötigten; für das 1. Lernjahr hingegen wurde nicht auf das Wiki zurückgegriffen, da dort im Unterricht kaum 
Texte behandelt wurden und der unterrichtete Wortschatz besser durch Videopodcasts und Videos erarbeitet werden konnte.

Durch die erwähnte Vorgangsweise, wonach die SchülerInnen wöchentlich den Wortschatz anhand der angeführten Medien bearbeiteten und diese allen SchülerInnen zur Verfügung gestellt wurden, lag der im Laufe des Italienisch-Unterrichts erarbeitete Wortschatz in allen Lernjahren beinahe vollständig in Form der oben genannten Medien vor. Die SchülerInnen wurden von der Autorin aufgefordert, diese zu verwenden und die Medien auch mobil z.B. über das Smartphone zu nutzen. D.h. die SchülerInnen konnten die produzierten Medien mit ihren mobilen Geräten sowie über den Computer abrufen und damit zum Lernen nutzen. Anzumerken ist, dass diese Medien von der Lehrperson im Regelunterricht nicht eingesetzt wurden.

Im nachfolgenden Kapitel werden nun ausgewählte Ergebnisse der durchgeführten Studie präsentiert. Hierbei liegt der Schwerpunkt, wie bereits erwähnt, auf den Unterschieden in den Vokabellernstrategien zwischen Experimentalund Kontrollgruppe sowie den untersuchten Lernjahren.

\section{Untersuchungsergebnisse}

Da die Erhebung direkt in der Schule vorgenommen wurde und daher auch den damit verbundenen Bedingungen unterworfen ist, verringerte sich die ursprüngliche Stichprobe $(\mathrm{N}=113)$ zunächst auf 111, da zwei Italienisch muttersprachliche SchülerInnen von dieser ausgenommen wurden. Durch Krankheit etc. reduzierte sich die ursprüngliche Stichprobe zusätzlich, da für die inferenzstatistische Auswertung nur die Datensätze jener SchülerInnen berücksichtigt wurden, die zu beiden Datenerhebungszeitpunkten anwesend waren.

Nach der Datenerhebung zu Schulbeginn und -ende wurden sämtliche Daten kodiert und in das Statistikprogramm SPSS (Version 21) eingegeben. Nach der Überprüfung möglicher Eingabefehler, der Normalverteilung der Daten und der Reliabilität erfolgte in weiteren Schritten die deskriptivstatistische sowie die inferenzstatistische Analyse. Innerhalb der Deskriptivstatistik wird die vorliegende

Barbara Hinger (Hg.), Zweite „Tagung der Fachdidaktik“ 2015.

Sprachsensibler Sach-Fach-Unterricht - Sprachen im Sprachunterricht.

(C) 2016 innsbruck university press, ISBN 978-3-903122-51-2, DOI 10.15203/3122-51-2 
Stichprobe anhand der Häufigkeit der Variablen beschrieben, während die Inferenzstatistik verallgemeinernde Aussagen über die Stichprobe hinaus auf die Grundgesamtheit macht (vgl. Raab-Steiner \& Benesch 2008: 11-13). Für die vorliegende Studie setzt sich die Stichprobe aus, wie bereits erwähnt, 111 SchülerInnen, zusammen, von denen auf die Grundgesamtheit, d.h. AHS-SchülerInnen, die Italienisch im schulischen Fremdsprachenunterricht lernen, geschlossen wird.

Für die inferenzstatistische Analyse fiel die Wahl auf den Mann-Whitney-UTest, weil dieser den Vergleich von zwei Gruppen, hier also von Experimentalund Kontrollgruppe, ermöglicht. Im vorliegenden Beitrag werden für Experimental- und Kontrollgruppe jeweils das erste und das dritte Lernjahr herangezogen. Mit dem Mann-Whitney-U-Test wurde bewusst ein nicht-parametrisches Verfahren gewählt, da sich dieses für nicht-normalverteilte Stichproben wie die vorliegende anbietet.

Der gesamte Fragebogen zu Schulbeginn, bestehend aus 73 Items (ohne die Angaben zur Person), weist einen Wert von 0,792 nach Cronbachs Alpha auf (vgl. Tab. 1), womit von einer reliablen Skala ausgegangen werden kann, da nach Mummendey \& Grau (2008: 102) für Einstellungsfragebögen Werte ab 0,80 als reliabel akzeptiert werden.

Tab. 1: Reliabilität gesamter Fragebogen (Schulbeginn)

\begin{tabular}{|c|c|}
\hline \multicolumn{2}{|c|}{ Realitätsstatistiken } \\
\hline Cronbachs Alpha & Anzahl der Items \\
\hline, 792 & 73 \\
\hline
\end{tabular}

Auch der am Ende des Schuljahres durchgeführte Fragebogen wurde einer Reliabilitätsanalyse unterzogen. Der gesamte Fragebogen befindet sich mit einem Cronbachs Alpha von 0,824 auch zu Schulende in einem adäquaten Reliabilitätsbereich (vgl. Tab. 2). 
Tab. 2: Reliabilität gesamter Fragebogen (Schulende)

\begin{tabular}{|c|c|}
\hline \multicolumn{2}{|c|}{ Realitätsstatistiken } \\
\hline Cronbachs Alpha & Anzahl der Items \\
\hline, 824 & 48 \\
\hline
\end{tabular}

Für die gesamte Stichprobe (N=111) kann ein durchschnittliches Alter von 15,84 festgestellt werden. Die L1 der SchülerInnen ist zu 91,3\% Deutsch; weitere Erstsprachen der SchülerInnen sind Bosnisch-Kroatisch-Serbisch, Japanisch, Koreanisch und Türkisch. Das Geschlecht der SchülerInnen verteilt sich mit 58,7\% auf männlich und 41,3\% auf weiblich und somit mit einer geringen Mehrheit zugunsten der Schüler.

\subsection{Deskriptivstatistische Ergebnisse}

Im Hinblick auf die schriftliche Befragung mittels Fragebogen werden nachfolgend ausgewählte Items der gesamten Stichprobe zu Schulbeginn und -ende einander gegenübergestellt. Die Auswahl der Items erfolgte im Hinblick auf deren Relevanz für die Forschungsfragen; d.h. die SchülerInnen wurden zu ihren bevorzugten Vokabellernstrategien befragt, um mögliche Unterschiede in deren Verwendung zwischen Experimental- und Kontrollgruppe sowie zwischen den Lernjahren auszumachen.

Für die gesamte Stichprobe lässt sich festhalten (vgl. Abb. 1), dass bezüglich neuer Medien ca. von einem Viertel der SchülerInnen Apps zum Vokabellernen herangezogen werden. Dass Sprachenlernen am besten mit dem Computer funktioniert, wird von 17,1\% befunden, wobei sich dieser Prozentsatz auf $15,0 \%$ zu Schulende verringert. Im Bereich der Strategien erfreut sich die sogenannte mündliche ,Zuhaltemethode` der größten Beliebtheit: Zu beiden Erhebungszeitpunkten gaben über 80,0\% der SchülerInnen an, die Vokabeln abzudecken und mündlich zu wiederholen. Auch die schriftliche ,Zuhaltemethode“ ist unter den SchülerInnen weit verbreitet: Jeweils ca. ein Drittel gibt an, die 
Wörter zu lernen, indem sie gelesen, abgedeckt und auswendig aufgeschrieben werden. Die geringste Zustimmung $(2,8 \%)$ zu Schulbeginn erfährt das Lernen mit Karteikärtchen, wobei hier eine starke Zunahme (auf 11,9\%) zu Schulende hin beobachtet werden kann. Damit lässt sich für die angeführten Items feststellen, dass für die gesamte Stichprobe so gut wie keine Änderungen zu beobachten sind. Einzig das Lernen mit Karteikärtchen weist eine Zunahme auf. Für die Fragestellung der hier dargestellten Studie lassen sich daraus noch keine Schlüsse ziehen, da bisher lediglich die Ergebnisse der gesamten Stichprobe, und nicht in Experimental- und Kontrollgruppe unterteilt, präsentiert wurden.

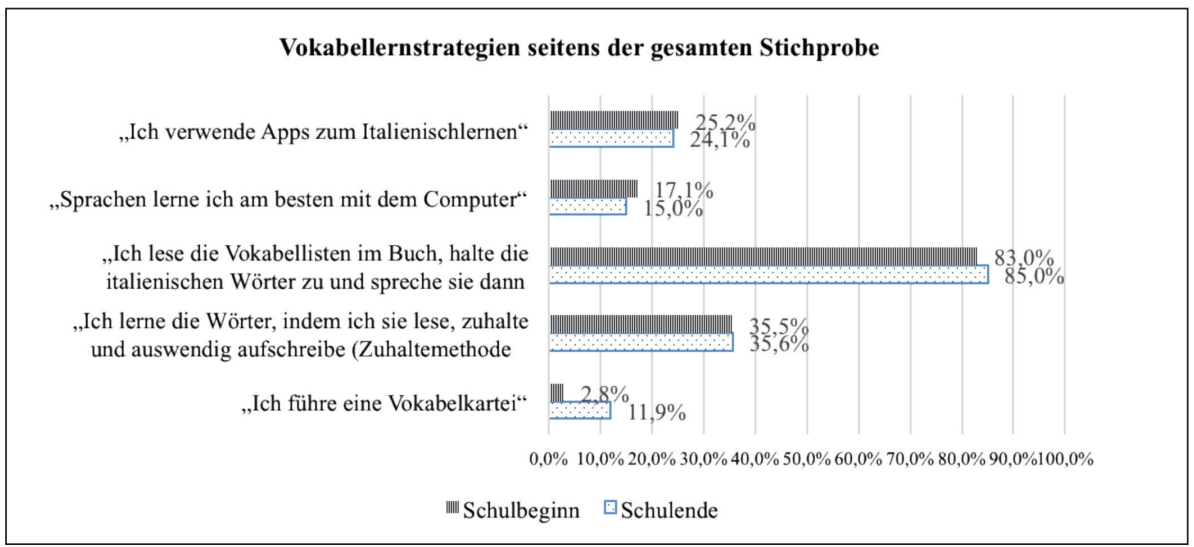

Abb. 1: Ergebnisse im Bereich Vokabellernstrategien; Deskriptivstatistik ausgewählter Items zu Schulbeginn und -ende

Im Rahmen der schriftlichen Befragung konnten die SchülerInnen u.a. subjektive Einschätzungen zum Vokabellernen auf einer Skala von 1 bis 10 angeben. Auch hier konnten, analog zu den soeben angeführten deskriptivstatistischen Ergebnissen, nur geringe Unterschiede in den Angaben zwischen Schulbeginn und -ende ausgemacht werden (vgl. Abb. 2). So liegt die Einschätzung, neue Medien sind sinnvoll zum Vokabellernen, mit einem Mittelwert von 5,75 bzw. 4,97 jeweils im mittleren Bereich. Die subjektive Wichtigkeit des Vokabellernens für die Beherrschung einer Fremdsprache ist hingegen - von einem anfänglichen Mittelwert von 8,73 auf 8,90 - leicht gestiegen. Der Wille, Italienisch-Vokabeln zu 
lernen, sinkt von einem Mittelwert von 5,21 auf 4,71. Die Überzeugung, sich mit den vorhandenen Vokabelkenntnissen in Italien, also im Zielland, verständigen zu können, nimmt innerhalb der gesamten Stichprobe am stärksten zu; nämlich von einem anfänglichen Mittelwert von 4,41 auf 5,45.

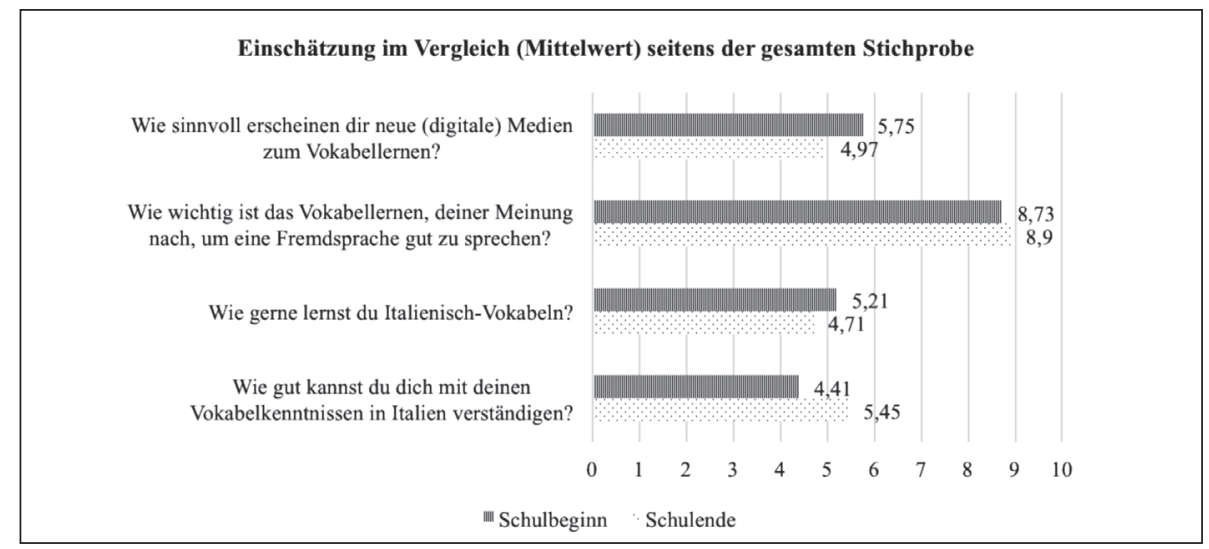

Abb. 2: Ergebnisse im Bereich Vokabellernstrategien; Deskriptivstatistik ausgewählter Items zu Schulbeginn und -ende

\subsection{Deskriptivstatistische Ergebnisse: Experimental- vs. Kontrollgruppe}

Für den vorliegenden Beitrag sollen, wie bereits erwähnt, die Ergebnisse der Experimental- und Kontrollgruppe miteinander verglichen werden, wobei beide Gruppen jeweils SchülerInnen aus dem ersten und dritten Lernjahr umfassen. Insgesamt kann dafür von einer teilnehmenden Stichprobe von 53 SchülerInnen ausgegangen werden, die an beiden Datenerhebungszeitpunkten anwesend waren. Mit einer Anzahl von 28 SchülerInnen für die Experimentalgruppe sowie 25 SchülerInnen für die Kontrollgruppe kann von zwei annähernd gleich großen Gruppen gesprochen werden, wobei auch die Anzahl der SchülerInnen pro Lernjahr - mit der Ausnahme des ersten Lernjahres der Kontrollgruppe mit 14 sehr ausgewogen ist (vgl. Tab. 3). 
Tab. 3: Experimental- vs. Kontrollgruppe (jeweils aus erstem und drittem Lernjahr bestehend)

\begin{tabular}{|c|c|c|c|c|}
\hline Schulstufe (Lernjahr) & \multicolumn{2}{|c|}{ Experimentalgruppe } & \multicolumn{2}{c|}{ Kontrollgruppe } \\
\hline & Anzahl & Prozent & Anzahl & Prozent \\
\hline 9. Schulstufe (1. Lernjahr) & 14 & $50,0 \%$ & 11 & $44,0 \%$ \\
\hline 11. Schulstufe (3. Lernjahr) & 14 & $50,0 \%$ & 14 & $56,0 \%$ \\
\hline & 28 & $100,0 \%$ & 25 & $100,0 \%$ \\
\hline
\end{tabular}

Nachfolgend sollen nun die beiden Gruppen im Hinblick auf die vier bereits in Kapitel 4.1. angeführten Items (vgl. Abb. 3) miteinander verglichen werden; die Ergebnisse werden in Abb. 4 visualisiert.

Für das erste Item „Wie gut kannst du dich mit deinen Vokabelkenntnissen in Italien verständigen?" kann festgehalten werden, dass sich zu Schulbeginn sowohl die Experimental- als auch die Kontrollgruppe gleich sicher in der Konversation im Zielland einschätzt, indem der Mittelwert für die Experimentalgruppe 4,25 und jener für die Kontrollgruppe 4,24 beträgt. Zu Schulende fühlt sich die Experimentalgruppe mit einem Mittelwert von 5,48 geringfügig sicherer in der

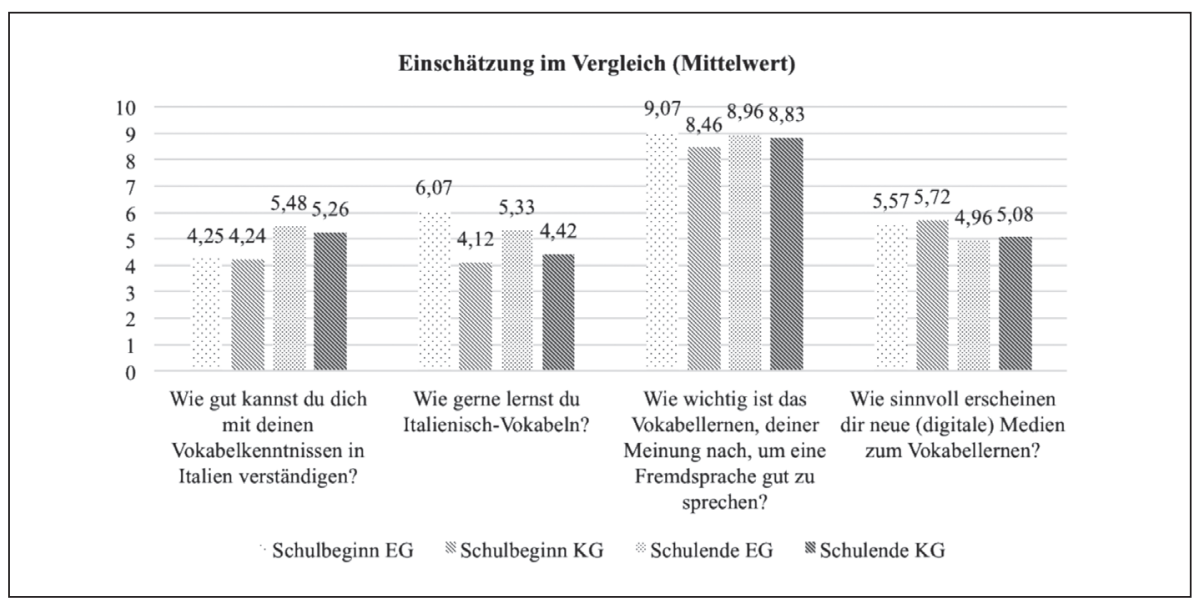

Abb. 3: Ergebnisse im Bereich Vokabellernstrategien: Experimental- (EG) vs. Kontrollgruppe (KG); Deskriptivstatistik ausgewählter Items zu Schulbeginn und -ende 
Konversation als die Kontrollgruppe mit einem Mittelwert von 5,26. Das heißt, beide Gruppen sehen einen Fortschritt in ihren Vokabelkenntnissen, wobei die Experimentalgruppe diesen geringfügig höher einschätzt.

Die Lust, Italienisch-Vokabeln zu lernen, ist innerhalb der Experimentalgruppe mit einem Mittelwert von 6,07 zu Schulbeginn höher als innerhalb der Kontrollgruppe mit einem Mittelwert von 4,12. Am Schulende sinkt die Lust der Experimentalgruppe am Lernen der Italienisch-Vokabeln (auf einen Mittelwert von 5,33), während diese in der Kontrollgruppe leicht steigt (auf einen Mittelwert von 4,42).

Die Wichtigkeit des Vokabellernens, um eine Fremdsprache gut sprechen zu können, wird von der Experimentalgrupe zu Schulbeginn sehr hoch eingeschätzt (Mittelwert von 9,07); auch für die Kontrollgruppe ist das Vokabellernen mit einem Mittelwert von 8,46 sehr bedeutsam. Während sich die Bedeutung des Vokabellernens für die Experimentalgruppe kaum verändert bzw. geringfügig sinkt (auf einen Mittelwert von 8,96), steigt dessen subjektive Relevanz in der Kontrollgruppe (auf einen Mittelwert von 8,83). Des Weiteren erachten die Experimental- und Kontrollgruppe zu Schulbeginn die Nutzung neuer Medien für das Vokabellernen als annähernd gleich wichtig (Mittelwert von 5,57 vs. 5,72). Am Schulende sinkt diese Einschätzung in beiden Gruppen, wobei der Mittelwert der Experimentalgruppe (auf 4,96) unwesentlich geringer abfällt als jener der Kontrollgruppe (auf 5,08).

Zusammenfassend kann gesagt werden, dass zwischen Experimental- und Kontrollgruppe zu den jeweiligen Datenerhebungszeitpunkten kaum Unterschiede bestehen: So schätzen beide Gruppen ihre Vokabelkenntnisse zu Schulbeginn und -ende annähernd gleich gut ein. Auch die Wichtigkeit des Vokabellernens, um eine Fremdsprache gut zu sprechen, wird von Experimental- und Kontrollgruppe zu beiden Zeitpunkten ähnlich wahrgenommen. Anders als angenommen, erscheinen der Experimentalgruppe neue Medien zu Schulende nicht sinnvoller zum Vokabellernen: Für beide Gruppen sind diese mittelmäßig sinnvoll fürs Vokabellernen. Nur bei der Lust, Italienisch-Vokabeln zu lernen, unterscheiden sich die beiden Gruppen: Die Experimentalgruppe lernt diese zu beiden Zeitpunkten lieber als die Kontrollgruppe; allerdings sinkt diese Lust innerhalb der Experimentalgruppe, während diese in der Kontrollgruppe geringfügig steigt.

Barbara Hinger (Hg.), Zweite „Tagung der Fachdidaktik“ 2015.

Sprachsensibler Sach-Fach-Unterricht - Sprachen im Sprachunterricht.

(C) 2016 innsbruck university press, ISBN 978-3-903122-51-2, DOI 10.15203/3122-51-2 
Inwieweit die beiden Gruppen signifikante Unterschiede in ihrer Einstellung zum Vokabellernen oder in den verwendeten Strategien aufweisen, zeigt das nachfolgende Kapitel, in dem inferenzstatistische Ergebnisse präsentiert werden.

\subsection{Inferenzstatistische Ergebnisse: Experimental- vs. Kontrollgruppe}

Um die bisherigen Aussagen zur Stichprobe um inferenzstatistische und damit über die Stichprobe hinausgehende Aussagen zu ergänzen, wurde mit dem UTest nach Mann \& Whitney ein nichtparametrisches Verfahren gewählt. Den Ausgangspunkt für die inferenzstatistische Analyse zu Schulende bildete die folgende Alternativhypothese: „Es gibt einen Unterschied in den verwendeten Vokabellernstrategien zwischen SchülerInnen, die den Wortschatz mittels digitaler Medien lernen, und jenen, die dies auf herkömmliche Art und Weise tun.“

Um die Vergleichbarkeit der beiden Gruppen im Hinblick auf die Vokabellernstrategien zu überprüfen, wurde der Mann-Whitney-U-Test auch zu Schulbeginn angewandt. Hier wurde die Alternativhypothese in elf Fällen (vgl. Tab. 4 und Tab. 5) bestätigt, womit sich die beiden Gruppen in den angeführten Items unterscheiden. So führt die Experimentalgruppe signifikant häufiger $(\mathrm{p}=0,002)$ ein Vokabelheft oder eine -mappe: Auf alle SchülerInnen der Experimentalgruppe trifft dies $\mathrm{zu}$, aber nur auf 80,0\% der Kontrollgruppe. Die Experimentalgruppe schreibt signifikant häufiger $(\mathrm{p}=0,010)$ die italienischen Wörter aus der Liste im Lehrbuch ab $(60,7 \%)$ sowie signifikant häufiger $(p=0,013)$ alle Vokabeln auf $(60,8 \%)$. Des Weiteren empfindet die Experimentalgruppe es signifikant hilfreicher $(\mathrm{p}=0,018)$, die italienischen Vokabelaufzeichnungen laut zu lesen (67,8\%), und hört signifikant häufiger $(p=0,020)$ täglich Musik mit dem mp3-Player $(92,9 \%)$. Schließlich geben die SchülerInnen der Experimentalgruppe signifikant häufiger $(\mathrm{p}=0,034)$ an, nur an einem bestimmten Ort gut zu lernen (65,4\%).

Die Kontrollgruppe hingegen empfindet die italienische Sprache sehr signifikant schwieriger $(p=0,000 ; 64,0 \%)$ und signifikant häufiger $(p=0,013)$ als nicht schön $(20,8 \%)$, woraus sich im Umkehrschluss festhalten lässt, dass innerhalb der Experimentalgruppe eine positivere Einstellung gegenüber der italienischen Sprache vorherrscht. Des Weiteren gibt die Kontrollgruppe signifikant häufiger $(p=0,048)$ an, die Vokabeln nur vor schriftlichen Überprüfungen (wie Test und

Barbara Hinger (Hg.), Zweite „Tagung der Fachdidaktik“ 2015.

Sprachsensibler Sach-Fach-Unterricht - Sprachen im Sprachunterricht.

(C) 2016 innsbruck university press, ISBN 978-3-903122-51-2, DOI 10.15203/3122-51-2 
Tab. 4: Inferenzstatistische Unterschiede zu Schulbeginn

\begin{tabular}{|c|c|c|c|c|c|}
\hline \multirow[t]{2}{*}{ Item } & \multicolumn{2}{|c|}{$\begin{array}{l}\text { Experimental- } \\
\text { gruppe }\end{array}$} & \multicolumn{2}{|c|}{ Kontrollgruppe } & \multirow{2}{*}{$\begin{array}{c}\text { Mann-Whitney-U- } \\
\text { Test } \\
\text { Signifikanz }\end{array}$} \\
\hline & Anzahl & $\begin{array}{l}\text { Gültige } \\
\text { Prozent }\end{array}$ & Anzahl & $\begin{array}{l}\text { Gültige } \\
\text { Prozent }\end{array}$ & \\
\hline $\begin{array}{l}\text { Ich führe ein/e Vokabelheft/- } \\
\text { mappe }\end{array}$ & 28 & $100,0 \%$ & 20 & $80,0 \%$ & $\mathrm{p}=0,002$ \\
\hline $\begin{array}{l}\text { Ich schreibe die italienischen } \\
\text { Wörter aus der Liste im Buch } \\
\text { ab }\end{array}$ & 17 & $60,7 \%$ & 5 & $20,0 \%$ & $\mathrm{p}=0,010$ \\
\hline Ich schreibe alle Vokabeln auf & 17 & $60,8 \%$ & 9 & $37,5 \%$ & $\mathrm{p}=0,013$ \\
\hline $\begin{array}{l}\text { Ich lese italienische Vokabelauf- } \\
\text { zeichnungen laut }\end{array}$ & 19 & $67,8 \%$ & 11 & $44,0 \%$ & $\mathrm{p}=0,018$ \\
\hline $\begin{array}{l}\text { Ich höre mit meinem mp3-Play- } \\
\text { er (iPod (C) täglich Musik }\end{array}$ & 26 & $92,9 \%$ & 16 & $64,0 \%$ & $\mathrm{p}=0,020$ \\
\hline $\begin{array}{l}\text { Ich lerne nur an einem be- } \\
\text { stimmten Ort gut }\end{array}$ & 17 & $65,4 \%$ & 8 & $34,8 \%$ & $\mathrm{p}=0,034$ \\
\hline $\begin{array}{l}\text { Ich finde die italienische Spra- } \\
\text { che schwierig }\end{array}$ & 4 & $14,3 \%$ & 16 & $64,0 \%$ & $\mathrm{p}=0,000$ \\
\hline $\begin{array}{l}\text { Ich finde die italienische Spra- } \\
\text { che ,unschön' }\end{array}$ & 1 & $3,6 \%$ & 5 & $20,8 \%$ & $\mathrm{p}=0,013$ \\
\hline $\begin{array}{l}\text { Ich lerne meine Vokabeln nur } \\
\text { vor Tests/Schularbeiten }\end{array}$ & 7 & $25,9 \%$ & 13 & $52,0 \%$ & $\mathrm{p}=0,048$ \\
\hline $\begin{array}{l}\text { Ich verwende im Unterricht ein } \\
\text { zweisprachiges Wörterbuch }\end{array}$ & 0 & $0,0 \%$ & 2 & $8,0 \%$ & $\mathrm{p}=0,050$ \\
\hline
\end{tabular}


Schularbeit) zu lernen (52,0\%). Ferner verwenden die SchülerInnen der Kontrollgruppe signifikant häufiger $(\mathrm{p}=0,050)$ ein zweisprachiges Wörterbuch im Unterricht $(8,0 \%)$. Abschließend soll angeführt werden, dass die Experimentalgruppe die Italienisch-Vokabeln signifikant $(p=0,002)$ lieber lernt als die Kontrollgruppe (vgl. Tab. 5; Mittelwert: 6,07 vs. 4,12).

Tab. 5: Inferenzstatistischer Unterschied bei Item „Wie gerne lernst du Italienisch-Vokabeln?“ zu Schulbeginn; Lagemaße

\begin{tabular}{|c|c|c|c|c|c|c|c|}
\hline \multirow{2}{*}{ Item } & \multicolumn{3}{|c|}{$\begin{array}{c}\text { Experimentalgruppe } \\
\text { (N=28) }\end{array}$} & \multicolumn{2}{c|}{ Kontrollgruppe (N=25) } & \multirow{2}{*}{$\begin{array}{c}\text { Mann- } \\
\text { Whitney- } \\
\text { U-Test }\end{array}$} \\
\cline { 2 - 7 } & $\begin{array}{c}\text { Mittel- } \\
\text { wert }\end{array}$ & Median & Modus & $\begin{array}{c}\text { Mittel- } \\
\text { wert }\end{array}$ & Median & Modus & \\
\hline $\begin{array}{l}\text { Wie gerne } \\
\text { lernst du } \\
\text { Italienisch- } \\
\text { Vokabeln? }\end{array}$ & 6,07 & 6,00 & 5 & 4,12 & 4,00 & 3 & $\mathrm{p}=0,002$ \\
\hline
\end{tabular}

Für die Alternativhypothese von großer Relevanz ist der U-Test nach Mann \& Whitney zu Schulende. Hier konnten drei signifikante Unterschiede zwischen Experimental- und Kontrollgruppe festgestellt werden. Demnach verwendet die Experimentalgruppe signifikant häufiger $(\mathrm{p}=0,007)$ ein zweisprachiges Wörterbuch im Unterricht: 42,9\% der Experimentalgruppe greifen auf ein zweisprachiges Wörterbuch zurück, allerdings nur 16,0\% der Kontrollgruppe. Des Weiteren schreibt die Experimentalgruppe signifikant häufiger $(\mathrm{p}=0,041)$ neue Vokabeln von der Tafel ab: auf 85,7\% der Experimentalgruppe und 76,0\% der Kontrollgruppe trifft dies zu. Schließlich führt die Experimentalgruppe signifikant häufiger $(p=0,022)$ ein Vokabelheft: $71,4 \%$ der Experimentalgruppe gibt an, dies zu tun, während dies nur für 36,0\% der Kontrollgruppe der Fall ist. 


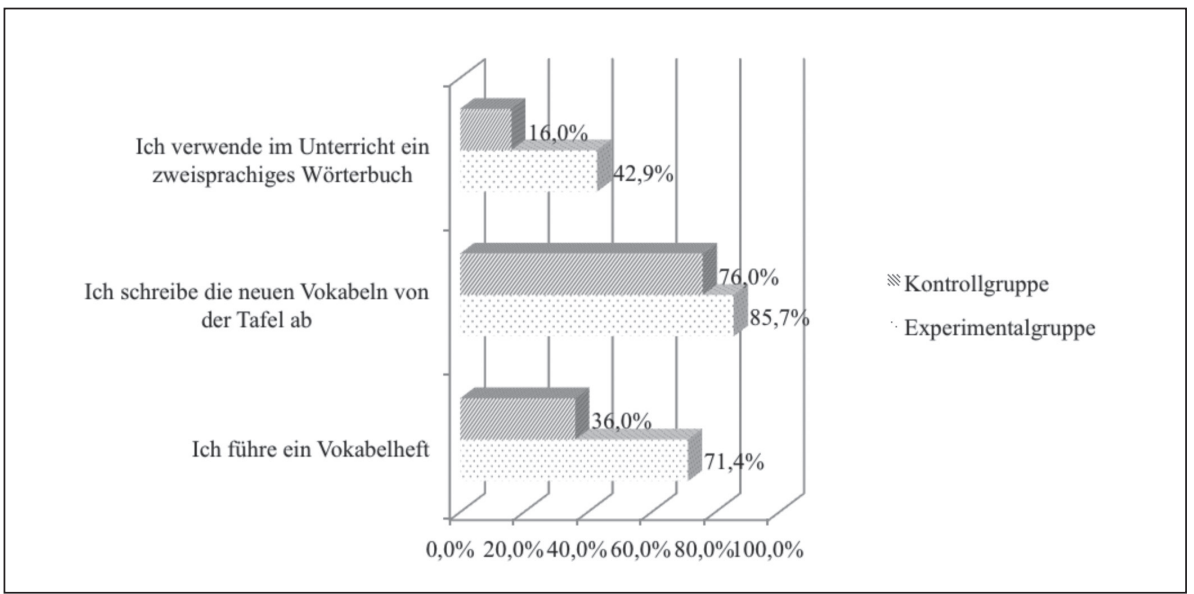

Abb. 4: Inferenzstatistische Unterschiede zu Schulende

Wie aus Abb. 4 hervorgeht, ist es jeweils die Experimentalgruppe, die den Aussagen in verstärktem Maße zustimmt. Da es sich bei den ermittelten Lernstrategien um traditionelle handelt, kann dies nicht direkt als Konsequenz des durchgeführten Projekts gewertet werden, denn vielmehr wäre ein vermehrtes Zurückgreifen auf moderne Medien zu erwarten. Zugunsten des Projekts kann angeführt werden, dass die Experimentalgruppe jedoch vermehrt Lernstrategien verwendet; in diesem Sinne kann von einer Bewusstseinsstärkung innerhalb der Experimentalgruppe ausgegangen werden.

\subsection{Korrelationen (Experimental- und Kontrollgruppe)}

Um Zusammenhänge zwischen den einzelnen Items festzustellen, wurde in einem weiteren Schritt die Rangkorrelation nach Spearman mittels des Statistikprogramms SPSS (Version 21) durchgeführt. Mögliche Zusammenhänge zwischen zwei Variablen können mittels eines Korrelationskoeffizienten berechnet werden. Dieser fungiert als Maßzahl für „die Stärke des Zusammenhangs“ (Bühl 2014: 426) und reicht von -1 bis +1 . Der als „r“ dargestellte Koeffizient weist auf einen starken Zusammenhang hin, wenn der Betrag nahe 1 angesiedelt ist und auf ei- 
nen schwachen bei einem Betrag nahe 0. Bei Werten bis 0,2 wird von einer sehr geringen Korrelation ausgegangen. Gering ist diese, wenn sie einen Wert bis 0,5 erreicht. Von einer mittleren Korrelation spricht man bei Werten bis 0,7, während Werte bis 0,9 eine hohe Korrelation anzeigen. Wird der Wert 0,9 überschritten, kann eine sehr hohe Korrelation angenommen werden (vgl. Bühl 2014: 426).

$\mathrm{Zu}$ Schulende konnten folgende mittlere Korrelationen für das Item „Sprachen lerne ich am besten mit dem Computer" eruiert werden.

Je sinnvoller neue Medien zum Vokabellernen von der Experimentalgruppe betrachtet werden, desto

- eher lernen die SchülerInnen Sprachen am besten mit dem Computer $(\mathrm{r}=0,526, \mathrm{p}=0,005)$.

- eher verwenden die SchülerInnen Wikis in der Freizeit ( $r=0,544, \mathrm{p}=0,003)$.

Je sinnvoller neue Medien zum Vokabellernen von den SchülerInnen der Kontrollgruppe betrachtet werden, desto

- eher geben die Lernenden an, Sprachen am besten mit dem Computer zu lernen $(r=0,547, p=0,005)$.

- wichtiger erscheint das Vokabellernen, um eine Fremdsprache gut sprechen zu können $(r=0,646, p=0,001)$.

Sowohl in der Experimental- als auch in der Kontrollgruppe lernen die SchülerInnen Sprachen am besten mit dem Computer, wenn sie neue Medien zum Vokabellernen sinnvoll erachten. Die Experimentalgruppe hingegen verwendet in der Freizeit eher neue Medien, je wertvoller diese auch zum Lernen gesehen werden; d.h. werden digitale Medien in der Freizeit verwendet, werden sie auch zum Lernen herangezogen. Bei den SchülerInnen der Kontrollgruppe nutzen jene SchülerInnen neue Medien zum Vokabellernen, die dem Vokabellernen für die Beherrschung einer Fremdsprache große Bedeutung beimessen. Daraus könnte man ableiten, dass SchülerInnen, die die Wichtigkeit des Wortschatzes für das Kommunizieren in einer Fremdsprache erkennen und damit vermutlich auch an einer erfolgreichen Kommunikation interessiert sind, zusätzlich mittels neuer Medien lernen. 


\subsection{Ausgewählte inferenzstatistische Ergebnisse: Signifikante Unterschiede zwischen dem ersten und dritten Lernjahr}

In einem zweiten Schritt wurde die Alternativhypothese „Es gibt einen Unterschied in den verwendeten Vokabellernstrategien zwischen SchülerInnen des ersten und dritten Lernjahres der Experimental- und Kontrollgruppe" aufgestellt und mittels H-Test nach Kruskal \& Wallis überprüft, der das Feststellen von Unterschieden zwischen mehr als zwei Gruppen erlaubt. Auch hier wurde das inferenzstatistische Verfahren zu Schulbeginn und -ende angewandt. Nachfolgend werden diese ermittelten Unterschiede nun - beginnend mit jenen zu Schulbeginn - präsentiert.

\section{Signifikante Unterschiede zu Schulbeginn}

Betrachtet man die signifikanten Unterschiede nach Lernjahren, lässt sich für das erste Lernjahr der Experimentalgruppe festhalten, dass diese SchülerInnen

- höchst signifikant häufiger $(\mathrm{p}=0,000)$ die italienischen (und deutschen) Wörter aus der Liste im Lehrbuch abschreiben.

- $\quad$ signifikant $(p=0,002)$ öfter ein/e Vokabelheft bzw. -mappe führen.

- sich signifikant $(\mathrm{p}=0,024)$ am häufigsten die Vokabeln von anderen abfragen lassen.

Die SchülerInnen des dritten Lernjahres der Experimentalgruppe

- können sich - gemäß eigener Einschätzung - mit den vorhandenen Vokabelkenntnissen signifikant $(\mathrm{p}=0,000)$ am besten in Italien verständigen.

- haben signifikant $(\mathrm{p}=0,007)$ am stärksten Lust, Vokabeln zu lernen.

- geben signifikant $(p=0,014)$ am häufigsten an, Vokabeln gerne zu lernen.

Das erste Lernjahr der Kontrollgruppe verwendet am signifikant häufigsten $(p=0,001)$ ein zweisprachiges Wörterbuch im Unterricht. 
Für das dritte Lernjahr der Kontrollgruppe gilt, dass die SchülerInnen

- die italienische Sprache signifikant $(\mathrm{p}=0,002)$ am häufigsten als schwierig empfinden.

- Italienisch signifikant $(\mathrm{p}=0,013)$ am häufigsten als nicht schön betrachten.

Signifikante Unterschiede zu Schulende

Das erste Lernjahr der Experimentalgruppe

- weist gemäß eigener Angaben die signifikant $(p=0,003)$ stärkste Verwendung von Podcasts in der Freizeit auf. Die SchülerInnen des ersten Lernjahres der Kontrollgruppe, des dritten Lernjahres der Experimentalgruppe und des dritten Lernjahres der Kontrollgruppe folgen in der Nutzung von Podcasts auf den nachfolgenden Plätzen.

- nutzt Weblogs in der Freizeit am signifikant $(\mathrm{p}=0,008)$ häufigsten. Danach folgen das erste Lernjahr der Kontrollgruppe, anschließend das dritte Lernjahr der Experimentalgruppe und jenes der Kontrollgruppe. Mit anderen Worten, es ist das erste Lernjahr, das diesem Medium stärker zugetan ist und hier vor allem das erste Lernjahr der Experimentalgruppe.

- schreibt zweispaltige Listen (Italienisch-Deutsch, Deutsch-Italienisch) am signifikant $(p=0,009)$ häufigsten. An nächster Stelle befindet sich das erste Lernjahr der Kontrollgruppe. Den dritten und vierten Platz nehmen das dritte Lernjahr der Experimentalgruppe und jenes der Kontrollgruppe ein.

Im dritten Lernjahr der Experimentalgruppe

- ist der Einsatz von zweisprachigen Wörterbüchern im Unterricht höchst signifikant $(p=0,000)$ verbreitet. An zweiter Stelle steht dieser Einsatz beim ersten Lernjahr der Kontrollgruppe, gefolgt vom ersten Lernjahr der Experimentalgruppe und vom dritten Lernjahr der Kontrollgruppe.

- ist das Führen eines Vokabelhefts am signifikant $(p=0,020)$ häufigsten verbreitet; am zweithäufigsten ist dieses im ersten Lernjahr der Experimentalgruppe. Innerhalb der Kontrollgruppe hingegen ist das Führen des Vokabelhefts im ersten Lernjahr häufiger als im dritten. 
Das erste Lernjahr der Kontrollgruppe

- verfasst signifikant $(\mathrm{p}=0,001)$ am häufigsten einen Beispielsatz mit dem zu lernenden Wort. Letzte Priorität räumt das dritte Lernjahr der Kontrollgruppe dieser Strategie ein. Innerhalb der Experimentalgruppe übt das dritte Lernjahr diese Strategie häufiger aus als das erste.

- erstellt signifikant $(p=0,004)$ am häufigsten einen Plan mit einer bestimmten Menge pro Tag zu lernender Vokabeln. An zweiter Stelle steht das erste Lernjahr der Experimentalgruppe. Am geringsten wird dies vom dritten Lernjahr der Kontrollgruppe durchgeführt. Damit scheint das erste Lernjahr - unabhängig von der Gruppe - häufiger einen Plan mit zu lernenden Vokabeln zu erstellen, wenn auch diese Strategie in der Kontrollgruppe signifikant stärker ausgeprägt ist.

- verwendet signifikant $(p=0,040)$ am häufigsten Apps zum Italienischlernen. Dahinter befindet sich das erste Lernjahr der Experimentalgruppe. Daran schließen das dritte Lernjahr der Experimentalgruppe und jenes der Kontrollgruppe an. Dieses Resultat entspricht damit nicht den vor der Studie erwarteten Ergebnissen, da der Anstieg von modernen Medien in der Experimentalgruppe erhofft wurde.

- führt signifikant $(\mathrm{p}=0,049)$ am häufigsten eine Vokabellernkartei. Das dritte Lernjahr der Kontrollgruppe setzt dies am geringsten um. Innerhalb der Experimentalgruppe ist die Vokabelkartei im ersten Lernjahr stärker verbreitet als im dritten.

- bildet signifikant $(\mathrm{p}=0,050)$ am häufigsten Sätze mit Wörtern, die ohne sprachlichen Kontext wenig Sinn enthalten (z.B. Adverbien). Im dritten Lernjahr der Experimentalgruppe ist dies am zweithäufigsten der Fall. Die Plätze drei und vier nehmen das erste Lernjahr der Experimentalgruppe und das dritte Lernjahr der Kontrollgruppe ein.

Für das dritte Lernjahr der Kontrollgruppe konnte für kein Item die signifikant höchste Zustimmung festgestellt werden.

Abschließend sollen nun die präsentierten Ergebnisse zusammengefasst und interpretiert werden. 


\section{Konklusion}

Der vorliegende Beitrag zeigte ausgewählte Ergebnisse einer sich über das Schuljahr 2012/13 erstreckenden Studie, in der das medienunterstützte Wortschatzlernen im schulischen Italienischunterricht im Zentrum steht. Im Unterschied zum Großteil bislang durchgeführter Studien, die sich v.a. mit dem Wortschatzerwerb des Englischen durch junge Erwachsene (zumeist Studierende) beschäftigen, stellt die vorliegende Untersuchung SchülerInnen in den Vordergrund und fokussiert die italienische Sprache. Damit versucht sie zwei Forschungsdesiderata entgegen zu wirken.

Die Versuchsanordnung der Studie bezieht eine Kontroll- und eine Experimentalgruppe ein, die sich jeweils aus SchülerInnen des ersten, dritten und vierten Lernjahres zusammensetzen. Dabei lernten die SchülerInnen der Experimentalgruppe den italienischen Wortschatz mittels selbst erstellter Medien, die gemäß dem mobilen Lernen beispielsweise mit den Smartphones genutzt werden können. Die SchülerInnen der Kontrollgruppe hingegen lernten den Wortschatz auf herkömmliche Weise. Es wurde davon ausgegangen, dass sich am Ende der Studie Unterschiede zwischen den beiden Gruppen bei den verwendeten Vokabellernstrategien zeigen. Wie der U-Test nach Mann \& Whitney erkennen lässt, unterscheiden sich die beiden Gruppen jedoch bereits zu Studienbeginn hinsichtlich der verwendeten Vokabellernstrategien und der Einstellungen zum Vokabellernen. So führt die Experimentalgruppe etwa vermehrt schriftliche Vokabelaufzeichnungen, während die Kontrollgruppe die italienische Sprache als ,schwierig' erachtet.

$\mathrm{Zu}$ Studienende zeigte sich in der Experimentalgruppe, vor allem im ersten Lernjahr, eine verstärkte Verwendung von Web 2.0-Tools (wie Wiki, Weblog, Podcast), was auf einen Bewusstmachungseffekt durch die Studie schließen lässt. Allerdings ist auch am Ende der Studie das Verfassen zweispaltiger Vokabellisten unter den SchülerInnen des ersten Lernjahres der Experimentalgruppe noch stark verbreitet, womit diese traditionelle Vorgangsweise beibehalten wurde. Für das dritte Lernjahr der Experimentalgruppe gilt es festzuhalten, dass die Lust, Italienisch-Vokabeln zu lernen, gegen Ende des Schuljahres sinkt und der Einsatz von traditionellen Medien zum Vokabellernen überwiegt. Damit kann für die

Barbara Hinger (Hg.), Zweite „Tagung der Fachdidaktik“ 2015.

Sprachsensibler Sach-Fach-Unterricht - Sprachen im Sprachunterricht.

(C) 2016 innsbruck university press, ISBN 978-3-903122-51-2, DOI 10.15203/3122-51-2 
Experimentalgruppe nur bedingt von einem Lernen an jenen Modellen, mit denen sie vertraut gemacht wurden und die sie auch nutzten, ausgegangen werden. Vielmehr scheint es für eine nachhaltige Änderung der Einstellung sowie der Vokabellernstrategien notwendig zu sein, den Unterricht selbst als Transformationsraum zu sehen und entsprechend auszurichten. In der vorliegenden Studie fertigten die SchülerInnen die Medien in ihrer Freizeit an, wozu eine hohe Motivation ihrerseits erforderlich war, die von den SchülerInnen auch aufgebracht wurde. Für eine nachhaltige Verwendung der genutzten Medien bedarf es jedoch vermutlich ebenfalls einer kontinuierlichen Begleitung im Unterricht. Dies erfordert unter anderem eine adäquate technische Ausstattung der Klassenzimmer, aber auch entsprechende Fortbildungsangebote für Lehrpersonen.

Bei den SchülerInnen der Kontrollgruppe konnte im Verlauf des Schuljahres für das erste Lernjahr ein quantitativer Zuwachs an Vokabellernstrategien beobachtet werden. Für das dritte Lernjahr wurde eine Reduktion der negativen Einstellung zur italienischen Sprache festgestellt. In Hirzinger-Unterrainer (i.V.) werden mögliche Beweggründe dafür diskutiert. Die hier vorgestellten Ergebnisse verweisen also darauf, dass die SchülerInnen die neuen Medien dann eher zum Lernen und in der Freizeit verwenden, je sinnvoller diese für das Lernen erachtet werden. Dennoch konnte in der Experimentalgruppe keine signifikante Steigerung des Medieneinsatzes konstatiert werden. Vielmehr ist es so, dass sowohl in der Experimental- als auch in der Kontrollgruppe die Verwendung von traditionellen Vokabellernstrategien vorherrschend bleibt. Umfangreichere Erklärungen für diese Präferenzen der SchülerInnen werden ebenfalls in HirzingerUnterrainer (i.V.) diskutiert.

Erste Ursachen dafür können in einer häufig genannten Aussage der SchülerInnen der Experimentalgruppe zum Medieneinsatz gefunden werden: „Ganz lustig aber viel Arbeit für wenige Vokabeln"; mit anderen Worten, der Arbeitsaufwand steht in den Augen der SchülerInnen nicht in einem ausgewogenen Verhältnis zum daraus gewonnenen Nutzen. Des Weiteren sind die traditionellen Strategien, wie das Lernen mit Vokabelheft, den SchülerInnen aus dem Schulalltag vertraut, was u.a. die folgende Aussage zeigt: „zusätzliche medien zum vokabelheft sind sehr gut. sie helfen dir mit ,eselsbrücken', aber ich würde es nicht als ersatz für mein vokabelheft benützen“. Insgesamt stellte das Projekt für die SchülerInnen

Barbara Hinger (Hg.), Zweite „Tagung der Fachdidaktik“ 2015.

Sprachsensibler Sach-Fach-Unterricht - Sprachen im Sprachunterricht.

(C) 2016 innsbruck university press, ISBN 978-3-903122-51-2, DOI 10.15203/3122-51-2 
dennoch eine willkommene Abwechslung dar: „Wir hatten sehr viel spaß [...]!“ Weiters soll angeführt werden, dass die SchülerInnen auch Vorteile beim Lernen mit neuen Medien sehen: „Ich finde diese Idee sehr gut, da ich finde dass man viel einfacher mit ihnen [neuen Medien] lernen kann.“

Abschließend kann festgehalten werden, dass die vorliegende Studie einen wichtigen Beitrag für die Fremdsprachendidaktik leistet, u.a. weil sie sich mit dem medialen Wortschatzlernen von Jugendlichen, und nicht von Erwachsenen, beschäftigt. Auch wenn die SchülerInnen weiterhin überwiegend traditionelle Vokabellernstrategien bevorzugen, zeigte sich dennoch die hohe Motivation der Lernenden, am Projekt teilzunehmen. Sowohl im ersten als auch im dritten Lernjahr der Experimentalgruppen kamen die SchülerInnen zum universitären Arbeitsplatz der Autorin, um den aktuell im Unterricht behandelten Wortschatz medial aufzubereiten. Da die SchülerInnen für die Teilnahme weder eine Note seitens der Lehrperson noch eine Bezahlung durch die Autorin erhielten, ist von einer intrinsischen Motivation auszugehen. Die mediale Aufbereitung des Wortschatzes wurde demnach als Abwechslung zum gewohnten Vokabellernen betrachtet, wodurch ein Ziel des Projekts erfüllt wurde. Mit Schmitt (2000: 142) kann zum gegenwärtigen Zeitpunkt resümiert werden, „[...] there is no 'right' or 'best' way to teach vocabulary“. Vielmehr scheint es so zu sein, dass SchülerInnen durch ein breiteres Angebot an Möglichkeiten, den Wortschatz zu erwerben, die für sie geeignetsten auswählen können. Das Lernen mit neuen Medien ist demnach - wie das Lernen mit herkömmlichen Medien auch - in unterschiedlichem Ausmaße für die einzelnen SchülerInnen hilfreich. Gerade die neuen Medien erleichtern es aber, den Wortschatz etwa zu visualisieren, was einigen SchülerInnen entgegenkommt. So erweitern neue Medien die Vielfalt an Alternativen, die SchülerInnen zum Lernen einsetzen können, wodurch auf deren individuelle Bedürfnisse noch besser eingegangen werden kann.

Für eine mögliche Wiederholung des Projekts könnte die aktive Partizipation der Lehrperson angedacht werden; oder falls durchführbar, das (Mit-)Unterrichten als ForscherIn und damit - etwa im Sinne der Aktionsforschung die Perspektive, den eigenen Fremdsprachenunterricht zu beforschen. Folglich würden die SchülerInnen bereits im Unterricht Erfahrungen mit neuen Medien sammeln, wodurch diese auch in der Freizeit häufiger zum Lernen herangezogen

Barbara Hinger (Hg.), Zweite „Tagung der Fachdidaktik“ 2015.

Sprachsensibler Sach-Fach-Unterricht - Sprachen im Sprachunterricht.

(C) 2016 innsbruck university press, ISBN 978-3-903122-51-2, DOI 10.15203/3122-51-2 
werden. Zudem könnte dadurch vermutlich sowohl die Medienkompetenz der Lehrperson als auch jene der SchülerInnen gesteigert werden. Weitere Studien könnten hier Einsicht bringen.

\section{Literatur}

Bortz, J. \& Döring, N. (2009). Forschungsmethoden und Evaluation. Für Human- und Sozialwissenschaftler. Mit 87 Tabellen (4., überarbeitete Aufl., Nachdruck). Heidelberg: Springer.

Bühl, A. (2014). SPSS 22: Einfübrung in die moderne Datenanalyse. Hallbergmoos: Pearson.

DIALANG (o.J.). Verfügbar unter: http://www.lancaster.ac.uk/researchenterprise/dialang/about [29.03.2016].

Dörnyei, Z. (2003). Questionnaires in second language research. Construction, administration, and processing. Mahwah, NJ: Erlbaum.

Hasegawa, K., Ishikawa, M., Shinagawa, N., Kaneko, K. \& Miyakoda, H. (2008). Learning Effects of Self-Made Vocabulary Learning Materials. In D. Sampson, G. Kinshuk, J. M. Spector, P. Isaías \& D. Ifenthaler (Hrsg.), Proceedings of the LADIS International Conference on Cognition and Exploratory Learning in Digital Age. Freiburg, Germany 13-15 October 2008 (153-159). Freiburg: International Association for Development of the Information Society.

Hirzinger-Unterrainer, E. M. (2014). Erwerb von lexikalischer Kompetenz. Wortschatz im Fremdsprachenunterricht. In S. Kapelari (Hrsg.), Tagung der Fachdidaktik 2013 (45-57). Innsbruck: innsbruck university press.

Hirzinger-Unterrainer, E. M. (i.V.). Medienunterstützter Wortschatzerwerb im Sprachenunterricht am Beispiel des Italienischen als Fremdsprache (Arbeitstitel). Habilitation, Universität Innsbruck, Innsbruck.

Hug, T. (2010). Mobiles Lernen. In K.-U. Hugger \& M. Walber (Hrsg.), Digitale Lernwelten (193211). Wiesbaden: Verlag für Sozialwissenschaften.

Intronati, Gianpaolo (1992). Italian Key Words: The Basic 2000 Word Vocabulary Arranged by Frequency, with Dictionaries. Cambridge: The Oleander Press.

Joseph, S., Binsted, K. \& Suthers, D. (2005). PhotoStudy: Vocabulary Learning and Collaboration on Fixed \& Mobile Devices. In Proceedings of 3rd IEEE International Workshop on Wireless and Mobile Technologies in Education (206-210).

Kennedy, C. \& Levy, M. (2008). L'italiano al telefonino. Using SMS to Support Beginners' Language Learning. ReCALL, 20 (3), 315-330. 
Laufer, B. \& Goldstein, Z. (2004). Testing Vocabulary Knowledge. Size, Strength, and Computer Adaptiveness. Language Learning, 54 (3), 399-436.

Macaro, E. (2010). Review of recent research (2000-2008) on applied linguistics and language teaching with specific reference to L2 Italian. Language Teaching, 43 (2), 127-153.

mpfs-Medienpädagogischer Forschungsverbund Südwest JIM-Studie (2015). Jugend, Information, (Multi-)Media. Basisuntersuchung zum Medienumgang 12- bis 19-Jähriger. Stuttgart: o.V. Verfügbar unter: http://www.mpfs.de/index.php?id=676 [29.03.2016].

Mummendey, H. D. \& Grau, I. (2008): Die Fragebogenmethode (5. Aufl.). Göttingen: Hogrefe.

Nation, I. S. P. (2011). Research into practice: Vocabulary. Language Teaching, 44 (4), 529-539.

Neveling, C. (2004). Wörterlernen mit Wörternetzen. Eine Untersuchung zu Wörternetæen als Lernstrategie und als Forschungsverfahren. Tübingen: Narr.

Porst, R. (2008). Fragebogen. Ein Arbeitsbuch. Wiesbaden: Verlag für Sozialwissenschaften.

Price, S. (2007). Ubiquitous computing: digital augmentation and learning. In N. Pachler (Hrsg.), Mobile learning: towards a research agenda (33-54). London: WLE Centre. Verfügbar unter: http:// www.londonmobilelearning.net/?page=Publications [29.03.2016].

Raab-Steiner, E. \& Benesch, M. (2008). Der Fragebogen. Von der Forschungsidee zur SPSS-Auswertung. Wien: facultas.wuv.

Read, J. (2000). Assessing Vocabulary. Cambridge: Cambridge University Press.

Schmitt, N. (2000). Vocabulary in language teaching. Cambridge: Cambridge University Press.

Schmitt, N. (2010): Key Issues in Teaching and Learning Vocabulary. In R. Chacón-Beltrán, C. Abello-Contesse \& M. Torreblanca-López (Hrsg.), Insights into Non-native Vocabulary Teaching and Learning (28-40). Bristol, Buffalo, Toronto: Multilingual Matters.

Stockwell, G. (2010). Using Mobile Phones for Vocabulary Activities. Examining the Effect of the Platform. Language Learning \& Technology, 14 (2), 95-110. Verfügbar unter: http://llt.msu.edu/ vol14num2/stockwell.pdf [29.03.2016].

Thornton, P. \& Houser, C. (2005). Using Mobile Phones in English Education in Japan. Journal of Computer Assisted Learning, 21 (3), 217-228.

Unterrainer, E. M. (2012). Mobiles Lernen im Fremdsprachenunterricht. Eine empirische Fallstudie zum Wortschatzerwerb mit Hilfe von digitalen Medien. Diplomarbeit, Universität Innsbruck. Innsbruck.

Wienold, K. (2004). Evaluation onlinebasierter Lebr-/Lernsysteme. Anforderung an Instrumente zur Evaluation Neuer Medien. Hamburg: Dr. Kovac.

Barbara Hinger (Hg.), Zweite „Tagung der Fachdidaktik“ 2015.

Sprachsensibler Sach-Fach-Unterricht - Sprachen im Sprachunterricht.

(C) 2016 innsbruck university press, ISBN 978-3-903122-51-2, DOI 10.15203/3122-51-2 
